\title{
Introduction du «European Exam in General Cardiology»
}

\author{
Felix C. Tannera, Michael J. Zellweger \\ a Prof. Dr med., Président de la commission pour la formation postgraduée et les examens de la Société Suisse de Cardiologie (SSC) \\ b Prof. Dr med., Président de la Société Suisse de Cardiologie (SSC)
}

Aux fins d'une meilleure lisibilité, on renoncera à l'utilisation simultanée de la forme masculine et féminine. Toutes les désignations concernant des personnes sont en conséquence valables autant pour des femmes que pour des hommes.

Le comité de la Société Suisse de Cardiologie (SSC) a décidé l'année passée de renouveler la partie écrite de l'examen de spécialiste en cardiologie. Le programme de formation postgraduée en cardiologie a été adapté en conséquence; ce dernier peut être consulté sur http://www.fmh.ch/bildung-siwf/fachgebiete/ facharzttitel-und-schwerpunkte/kardiologie.html.

Le renouvellement de la partie écrite de l'examen de spécialiste est justifié pour les raisons suivantes:

1. Les questions à choix multiple (QCM) générées à partir de plusieurs supports pédagogiques ne répondent plus aux exigences d'un examen, car la liste de questions était trop petite, n'était pas assez souvent adaptée et n'était pas systématiquement analysée quant à l'aptitude.

2. Le European Exam in General Cardiology (EEGC) a été développé et introduit par la European Society of Cardiology (ESC) en collaboration avec des associations professionnelles nationales, et ce afin de vérifier que les collègues en formation postgraduée / en formation disposent des connaissances

2016

2017

Ancienne forme d'examen

Nouvelle forme d'examen
2016

Partie pratique:

comme jusqu'ici en octobre

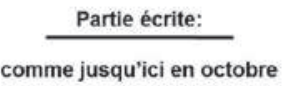

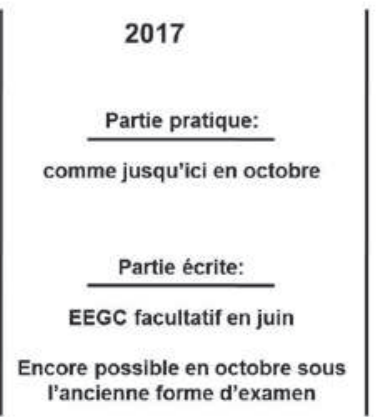

2018

Partie pratique:

comme jusqu'ici en octobre

Partie écrite:

EEGC obligatoire en juin

Plus possible sous 'ancienne forme d'examen spécifiques que l'on attend d'un médecin spécialisé* en cardiologie récemment qualifié. Ceci est cohérent avec l'objectif que la SSC poursuit concernant la partie écrite de l'examen de spécialiste.

3. Les Guidelines de la ESC sont déterminantes pour l'activité quotidienne cardiologique en Suisse et sont soutenues par la SSC. L'EEGC suit également les Guidelines de l'ESC.

4. L'EEGC dispose d'une liste de questions régulièrement évaluées, étendues et renouvelées en collaboration avec les sociétés nationales.

5. Outre la vérification des connaissances de base nécessaires, l'EEGC permet de plus d'évaluer les connaissances des candidats en provenance de la Suisse par comparaison transversale avec ceux du reste de l'Europe.

6. Compte tenu de la mobilité croissante de la population, l'EEGC est dès à présent utile et gagnera encore en importance à l'avenir (éventuellement dans l'esprit d'un «European Cardiologist»).

Contrairement à la partie écrite, la partie pratique de l'examen de spécialiste est maintenue telle quelle. Les aptitudes et les compétences pratiques ne peuvent pas être vérifiées par un examen QCM et doivent, par conséquent, continuer à être testées à partir de cas concrets et auprès du patient.

Le passage de l'ancien examen écrit au nouvel examen écrit se déroulera comme suit (illustration):

- En 2016, la partie écrite de l'examen de spécialiste se déroulera sans changement. La participation à l'EEGC n'est pas encore possible pour les candidats en provenance de Suisse.

- Une solution de transition est prévue pour 2017. Elle consiste à faire passer en parallèle l'EEGC et l'examen écrit sous sa forme ancienne. Les candidats peuvent participer à l'EEGC; c'est ce que souhaite la SSC, sans toutefois être obligatoire. Si un candidat réussit l'EEGC, qui a lieu en juin, il est automatiquement admis à se présenter à l'examen pratique. S'il ne réussit pas l'EEGC, il peut passer l'examen écrit sous sa forme ancienne en octobre. L'examen pra- 
tique se déroule, comme c'était le cas jusqu'à présent, le lendemain de l'examen écrit, sous forme inchangée.

- A partir de 2018, il est prévu d'utiliser exclusivement l'EEGC comme examen écrit. Ceci signifie que les candidats doivent passer l'examen écrit en juin, comme c'était le cas jusqu'à présent. Mais les candidats doivent désormais avoir réussi l'examen écrit pour pouvoir se présenter à l'examen pratique. Dans ce contexte, il est recommandé de passer l'examen écrit dès la $3^{\mathrm{e}}$ année de la formation de spécialiste, mais en revanche de ne passer l'examen pratique que vers la fin de la formation de spécialiste.

Les supports pédagogiques suivants sont recommandés pour la préparation à l'EEGC:

- ESC Textbook of Cardiovascular Medicine

- Guidelines de l'ESC et de l'ACC/AHA

- Supports pédagogiques en ligne

- ESC eLearning: http://www.escardio.org/

- ACC Cardiosource: http://www.acc.org/

- Education in Heart: http://heart.bmj.com/site/ about/education.xhtml

Prof. Dr med. Felix C. Tanner Directeur adjoint du service de cardiologie Médecin chef de service en échocardiographie Universitäres Herzzentrum Zürich

Rämistrasse 100

CH-8091 Zurich

L'EEGC se déroule en langue anglaise sous forme d'examen QCM et il peut être passé en juin, soit dans un lieu en Suisse alémanique ou en Suisse romande. La corresmais la partie pratique seulement en octobre,

\section{Informations importantes pour les} candidats qui participent à I'EEGC 2017

- Inscription jusqu'au 15.10.2016 à info[at]swisscardio.ch

- ESCTextbook of Cardiovascular Medicine en cadeau après l'inscription

pondance avec les candidats passe exclusivement par le secrétariat de la SSC; l'ESC n'entrera pas directement en contact avec les candidats. La taxe pour l'EEGC est contenue dans la taxe d'examen prélevée par la SSC pour l'ensemble de l'examen de spécialiste.

La SSC apprécierait que le plus grand nombre possible de candidats participent à l'EEGC dès 2017. L'inscription à l'EEGC 2017 doit parvenir au secrétariat de la SSC d'ici le 15 octobre 2016. L’EEGC 2017 aura lieu le 15 juin 2017. Les candidats qui s'inscrivent à l'EEGC 2017 recevront chacun un ESC Textbook of Cardiovascular Medicine en cadeau de la part de la SSC pour se préparer à l'examen.

Le comité de la SSC est convaincu que cette adaptation de l'examen de spécialiste améliorera la qualité de l'examen et contribuera à multiplier les interactions entre la relève en cardiologie et les activités de l'ESC, en particulier concernant les Guidelines et les possibilités de formations postgraduées.
Crédit photo

mis à disposition par les auteurs 